\section{A young adult with a fulminant Staphylococcus aureus infection and multiple thrombi}

\author{
Eric Martin, Wesam Frandah, Rishi Raj, \\ Kenneth Nugent \\ Department of Internal Medicine, Texas \\ Tech University Health Sciences Center, \\ Lubbock, TX, USA
}

\begin{abstract}
Community-acquired methicillin-resistant Staphylococcus aureus (CA-MRSA) can cause skin and soft tissue infection and necrotizing pneumonia. We report a patient who developed ankle cellulitis following minor trauma and developed MRSA bacteremia, necrotizing pneumonia, respiratory failure, shock, and multiple thrombi. This case highlights the fulminant course, the possible need for empiric antibiotic coverage on admission for MRSA, and the high likelihood of thrombotic complications in these patients.
\end{abstract}

\section{Introduction}

Community-acquired methicillin-resistant Staphylococcus aureus (CA-MRSA) infections can present with necrotizing pneumonia and progress rapidly to respiratory failure and multiorgan failure. Pediatric cases with MRSA osteomyelitis often have deep venous thrombosis. ${ }^{1,2}$ We report a patient who had minor ankle trauma complicated by CA-MRSA pneumonia, respiratory failure, shock, and multiple thrombi.

\section{Case Report}

A 32-year-old woman presented to the emergency department (ED) with shortness of breath, hemoptysis, and right leg swelling for four days. Four days prior to presentation, the patient reported rolling her ankle off a curb. One day after this event she noted diffuse muscle pain followed by fever and chills, cough with blood-tinged sputum, and persistent right lower extremity pain and swelling. Social history included 15 pack-years smoking and inhalational cocaine abuse. The patient had no children and was not on oral contraceptives.

In the ED, her vital signs included 28 breaths per minute, 115 heart beats per minute, a normal blood pressure, a normal temperature, and an oxygen saturation of $89 \%$ on room air. Lung examination revealed bronchial breath sounds with egophony over the left upper zone. Cardiovascular and abdominal examinations were within normal limits. She had erythema and edema of the right lower leg and pain on dorsiflexion of the right foot. She had no peripheral signs of intravenous drug use.

Initial lab investigation included WBC of $3.6 \times 10^{9} / \mathrm{L}$, hemoglobin of $10.7 \mathrm{~g} / \mathrm{dL}$, platelet count of $125 \times 10^{9} / \mathrm{L}$, prothrombin time of $15.7 \mathrm{~s}$, partial thromboplastin time of $35 \mathrm{~s}$, a protein $\mathrm{C}$ level of $38 \%$ of the reference range (normal 70 to $180 \%$ ), BUN of $33 \mathrm{mg} / \mathrm{dL}$, and creatinine of $2.4 \mathrm{mg} / \mathrm{dL}$. The initial arterial blood gas revealed a $\mathrm{pH}$ of 7.27 , a $\mathrm{PaCO}_{2}$ of $35.2 \mathrm{mmHg}$, and a $\mathrm{PaO}_{2}$ of $62.3 \mathrm{mmHg}$ on a $\mathrm{FiO}_{2}$ of $40 \%$. Chest $\mathrm{x}$-ray revealed bilateral infiltrates (Figure 1). A Doppler ultrasound of the lower extremities revealed a right leg deep vein thrombosis (DVT) that propagated into the distal popliteal vein. Urine drug screen was positive for cocaine. Computed tomography (CT) scan of the chest showed multiple patchy infiltrates with the largest in the anterior segment of the left upper lobe (Figure 2); a CT angiogram was negative for pulmonary emboli.

Initial management included intravenous fluids, oxygen by mask, empiric antibiotic therapy with levofloxacin and ceftriaxone, and therapeutic enoxaparin. The orthopedic consultant concluded that there were no signs of necrotizing soft tissue infection, osteomyelitis, or septic arthritis. After seven hours, her breathing became more labored, and her blood pressure dropped. She underwent urgent intubation and was started on norepinephrine. Ultrasound of the abdomen to evaluate a possible right upper quadrant mass noted absent flow in the superior mesenteric, splenic, and main portal vein and its branches consistent with splanchnic thromboses. Eight hours after admission to the intensive care unit vasopressin and stress dose hydrocortisone were started. Twelve hours after admission empiric vancomycin therapy was started. Eighteen hours after admission, the patient developed ventricular tachycardia and died after prolonged resuscitation efforts. Methicillin-resistant Staphylococcus aureus was eventually grown from both the sputum and blood cultures. An assay for PVL or other staphylococcal toxins was not obtained, but her clinical course is consistent with a hypervirulent staphylococcal infection.

\section{Discussion}

This patient was young and healthy. Both the CURB65 and pneumonia severity index predicted a low mortality rate. However, she had a fulminant staphylococcal infection with
Correspondence: Kenneth Nugent, Department of Internal Medicine, Texas tech University health Sciences Center, 3601 4th Street, Lubbock, TX 79430, USA.

Tel. +806.743-3155 - Fax: +806.743-3148. E-mail: kenneth.nugent@ttuhsc.edu

Key words: necrotizing pneumonia, MRSA, Panton-Valentine toxin, deep venous thrombosis.

Contributions: the authors contributed equally.

Received for publication: 14 September 2011. Revision received: 3 November 2011.

Accepted for publication: 28 October 2011.

This work is licensed under a Creative Commons Attribution NonCommercial 3.0 License (CC BYNC 3.0).

(C) Copyright E. Martin et al., 2011

Licensee PAGEPress, Italy

Chest Disease Reports 2011; 1:e16

doi:10.4081/cdr.2011.e16

cellulitis, bacteremia, necrotizing pneumonia, acute respiratory failure, and multiple deep venous thrombi. Staphylococcal lung infections usually develop after viral infections of the respiratory tract, which allows staphylococcal adherence to epithelial surfaces and invasion. Gillet and coworker reported on 16 patients with necrotizing pneumonia secondary to Staphylococcus aureus strains producing the Panton-Valentine leukocidin (PVL). ${ }^{3}$ These patients were young (median age 15) and healthy. Most (75\%) had an influenza-like syndrome prior to presentation. They presented with high temperatures, tachycardia, tachypnea, cyanosis, and hypotension. Thirty-eight percent had hemoptysis. The median trough WBC was $1.8 \mathrm{kU} / \mathrm{L}$, and the median trough platelet count was $70 \mathrm{kU} / \mathrm{L}$. Most (86\%) required mechanical ventilation, and the mortality rate was $75 \%$ even though none had an underlying disorder. Most of the bacterial isolates were methicillin-sensitive. Autopsies revealed massive ulceration of the tracheobronchial tree with adherent staphylococci and alveolar hemorrhage and necrosis with large clusters of staphylococci. A recent literature review reported that PVL positive communityacquired pneumonia also occurs in patients with methicillin-resistant Staphylococcus aureus (MRSA), and these patients often have influenza-like symptoms, complicated hospital courses with acute respiratory failure and multiorgan system failure, and high mortality (50\%). ${ }^{4}$

Staphylococcal pneumonia can also develop during episodes of staphylococcal bacteremia. Our patient likely developed cellulitis following minor ankle trauma, which resulted in bacteremia. Community-acquired methicillin- 
resistant Staphylococcus aureus (CA-MRSA) infections often involve the skin and soft tissues and then visceral organs such as lung and bone. Swaminathan reported a case with staphylococcal skin abscesses complicated by septic arthritis, multiple metastatic bone and soft tissue infections, and cavitary pneumonia secondary to a community-acquired methicillin-sensitive Staphylococcus aureus infection which was PVL positive. ${ }^{5}$ These authors suggested that their patient had the PVL syndrome characterized by necrotizing pneumonia, severe soft tissue and bone infection, and DVT. These patients are typically young and healthy and may or may not have a history of skin infection. The DVT presumably develops as a consequence of venous injury related to the adjacent cellulitis. Our patient meets the definition for this syndrome but also had venous thrombi remote from the lower extremity site of inflammation, which suggests that these infections can cause systemic hypercoagulability. Vardakas's literature review demonstrated that DVT is relatively frequent in these patients even without cellulitis and has a strong association with mortality. In this study $74 \%$ of patients (29/39) who died had DVT. ${ }^{4}$ Two recent publications have described children with staphylococcal osteomyelitis and deep venous thrombosis., ${ }^{1,2}$ Most of these thrombi occurred in the proximal leg veins but some were at remote sites including the inferior vena cava, superior vena cava, and left brachiocephalic vein. ${ }^{2}$ However, Lin et al. have

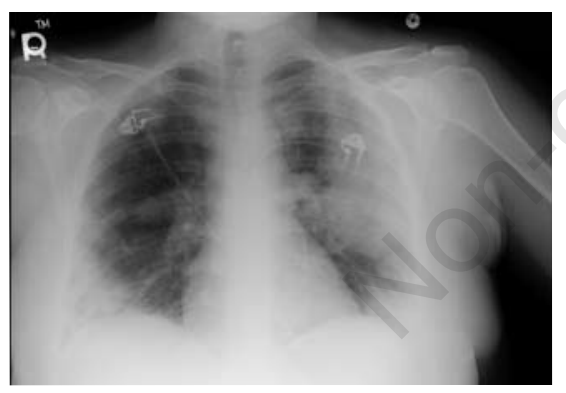

Figure 1. Plain chest $\mathrm{X}$-ray reveals consolidation in the left lung and opacities in the right base.

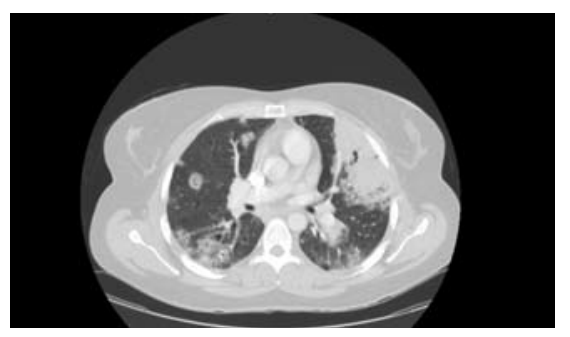

Figure 2. Computed tomography of the chest reveals bilateral infiltrates with areas of necrosis. described four adult patients with septic pulmonary emboli, bacteremia, and deep tissue infection. ${ }^{6}$ These patients didn't have deep venous thrombi, and, therefore, DVT is not a consistent complication of these infections. Splanchnic vein thrombosis often occurs in patient with cirrhosis, inflammatory disorders in the abdomen, abdominal trauma and hypercoagulable states. ${ }^{7,8}$ Our patient did not appear to have any other contributing factors beyond her acute staphylococcal infection. However, it is possible that she had an underlying hypercoagulable disorder such as antiphospholipid syndrome, which was triggered by ankle trauma and cellulitis and contributed, to her clinical course.

The Panton-Valentine leukocidin is a bicomponent protein secreted by some staphylococcal strains. This toxin binds to leukocyte membranes and creates pores. ${ }^{9}$ At high concentrations it causes cell lysis with the release of toxic enzymes, chemotactic factors, and reactive oxygen species. It can cause severe inflammation when injected into rabbit skin and pneumonia when inoculated into rabbit lungs. There are high concentrations of PVL in human skin abscesses caused by PVL positive bacteria. At low concentrations it causes leukocyte apoptosis. Both results cause leukopenia and potentially reduce $\mathrm{WBC}$ numbers at sites of infection. PVL-mediated leukocyte lysis could contribute to venous damage in clinical syndromes like sepsis with low flow states and endothelial activation. Epidemiological studies report that PVL has a strong association with virulent CA-MRSA infections, and animal studies provide potential explanations. However, some experimental studies in animals, especially mice, suggest that virulence also reflects other toxins. These would include alpha-toxin which lyses macrophages and lymphocytes and has other proinflammatory effects and phenolsoluble modulin, a surfactant-like peptide, which lyses neutrophils. ${ }^{10-12}$ These toxins may have additive or synergistic effects in CAMRSA infections. In addition, virulence might reflect overexpression of toxin production rather than a particular toxin effect. Staphylococcal regulatory genes control toxin production, and this process depends on bacterial density and mutations in these genes.

The mortality rate is approximately $50 \%$ in CA-MRSA necrotizing pneumonia, and most patients require mechanical ventilation. The Infectious Disease Society of America guidelines published in 2011 recommends empiric coverage for MRSA in patients with community-acquired pneumonia if they require ICU admission, have necrosis, or have empyema. This represents a AIII recommendation based on expert opinion. ${ }^{13}$ Vancomycin is the first line treatment and should be used in doses to achieve trough levels of $15-20 \mu \mathrm{g} / \mathrm{mL}$. However, after a positive culture for MRSA is identified, it is important to determine the vancomycin minimum inhibitory concentration for that particular isolate. If the MIC is near the breakpoint for susceptibility $(2 \mu \mathrm{g} / \mathrm{mL})$ or the clinical picture deteriorates, linezolid should be used. In addition, antimicrobials that suppress exotoxin production and secretion should be considered. Clindamycin decreases staphylococcal exotoxin production in vivo and in vitro, and some authors think that the initial therapy should include linezolid and clindamycin. ${ }^{14}$ A D-test should be performed to rule out inducible clindamycin resistance. In very sick patients with multiorgan failure or shock, intravenous immunoglobulin might be considered since it neutralizes PVL in vitro ${ }^{15,16}$ It has been used in a small number of cases at doses of $2 \mathrm{gm}$ per kilogram with a second dose at 48 hours. Thrombi should be treated with therapeutic doses of heparin. Household contacts of patients with skin infections should be evaluated for infection.

In summary, CA-MRSA can cause both skin and soft tissue infections and fulminant necrotizing pneumonia. Staphylococci with the PVL genotype have a strong epidemiological association with virulent infections. This toxin causes leukocyte lysis at high concentrations and leukocyte apoptosis at low concentrations. Both effects cause leukopenia and probably contribute to the virulence of the bacteria. The PVL syndrome includes necrotizing pneumonia, skin infection, and deep venous thrombosis. Treatment should include vancomycin or linezolid. The addition of clindamycin to inhibit toxin production and IgG to bind toxins should be considered. Patients should be evaluated carefully and perhaps repeatedly for DVT. Our patient had a fulminant course, and it is unlikely that earlier administration of vancomycin would have changed the outcome. However, she provides an important lesson for clinicians treating patients with pneumonia.

\section{References}

1. Gonzalez BE, Teruya J, Mahoney DH, et al. Venous thrombosis associated with staphylococcal osteomyelitis in children. Pediatrics 2006;117:1673-9.

2. Crary SE, Buchanan GR, Drake CE, et al. Venous thrombosis and thromboembolism I children with osteomyelitis. J Pediatr 2006;149:537-41.

3. Gillet Y, Vanhems P, Lina G, et al. Factors predicting mortality in necrotizing community-acquired pneumonia caused by Staphylococcus aureus containing PantonValentine leukocidin. Clin Infect Dis 2007;45:315-21.

4. Vardakas KZ, Matthaiou DK, Falagas ME. Comparison of community-acquired pneu- 
monia due to methicillin-resistant and methicillin-susceptible Staphylococcus aureus producing the Panton-Valentine leukocidin. Int $\mathrm{J}$ Tuberc Lung Dis 2009;13;1476-85.

5. Swaminathan A, Massasso D, GotisGraham I, Gosbell I. Fulminant methicillinsensitive Staphylococcus aureus infection in a healthy adolescent, highlighting 'Panton-Valentine leukocidin syndrome'. Intern Med 2006;36:744-7.

6. Lin,MY, Rezai K, Schwartz DN. Septic pulmonary emboli and bacteremia associated with deep tissue infections caused by community-acquired methicillin-resistant Staphylococcus aureus. J Clin Micrbiol 2008; 46:1553-5.

7. Ponziani FR, Zocoo MA, Campanale C, et al. Portal vein thrombosis: insight into physiopathology, diagnosis, and treatment. World J Gastroenterol 2010;16:143-55.

8. Gertsch P, Matthews J, Lerut J, et al. Acute thrombosis of the splanchnic veins. Arch
Surg 1993;128:341-5.

9. Boyle-Vavra S, Daum RS. Communityacquired methicillin-resistant Staphylococcus aureus: the role of PantonValentine leukocidin. Lab Invest 2007;87:39.

10. Liles WC, Thomsen AR, O'Mahony S, Klebanoff SJ. Stimulation of human neutrophils and monocytes by staphylococcal phenol-soluble modulin. J Leuk Biol 2001;70:96-102.

11. Hongo I, BabaT, Oishi K, et al. PhenolSoluble Modulin $\alpha 3$ Enhances the Human Neutrophil Lysis Mediated by PantonValentine Leukocidin. $\mathrm{J}$ Infect Dis 2009;200:715-23.

12. Konig B, Prevost G, Piemont Y, Konig W. Effects of Staphylococcus aureus leukocidins on inflammatory mediator release from human granulocytes. J Infect Dis 1995;171:607-13.

13. Liu C, Bayer A, Cosgrove SE, et al. Clinical practice guidelines by the Infectious
Disease Society of America for the treatment of methicillin- resistant Staphylococcus aureus infections in adults and children. Clin Infect Dis 2011;52:1-38.

14. Stevens DL, Ma Y, Salmi DB, et al. Impact of antibiotics on expression of virulenceassociated genes in methicillin-sensitive and methicillin- resistant Staphylococcus aureus. J Infect Dis 2007;195:202-11.

15. Gauduchon V, Cozon G, Vandenesch F, et al. Neutralization of Staphylococcus aureus Panton Valentine leukocidin by intravenous immunoglobulin in vitro. $\mathrm{J}$ Infect Dis 2004;189:346-53.

16. Hampson FG, Hancock SW, Primhak RA. Disseminated sepsis due to a PantonValentine leukocidin producing strain of community acquired meticillin resistant Staphylococcus aureus and use of intravenous immunoglobulin therapy. Arch Dis Child 2006;91:201. 